\title{
Situation Awareness Levels to Evaluate the Usability of Augmented Feedback to Support Driving in an Unfamiliar Traffic Regulation
}

\author{
Hasan J. Alyamani ${ }^{1}$, Ryan Alturki ${ }^{2}$, Arda Yunianta ${ }^{3}$, Nashwan A. Alromema ${ }^{4}$, \\ Hasan Sagga ${ }^{5}$, Manolya Kavakli ${ }^{6}$ \\ Faculty of Computing and Information Technology in Rabigh, King Abdulaziz University, Jeddah, Saudi Arabia ${ }^{1,3,4}$ \\ College of Computer and Information Systems, Umm Al-Qura University, Makkah, Saudi Arabia ${ }^{2}$ \\ College of Business, University of Jeddah, Asfan, Jeddah, Saudi Arabia ${ }^{5}$ \\ Faculty of Science and Engineering, Macquarie University, Sydney, Australia ${ }^{6}$
}

\begin{abstract}
Driving in an unfamiliar traffic regulation using an unfamiliar vehicle configuration contributes to increase number of traffic accidents. In these circumstances, a driver needs to have what is referred to as 'situation awareness' (SA). SA is divided into (level 1) perception of environmental cues, (level 2) comprehension of the perceived cues in relation to the current situation and (level 3) projection of the status of the situation in the near future. On the other hand, augmented feedback (AF) is used to enhance the performance of a certain task. In Driving, AF can be provided to drivers via in-vehicle information systems. In this paper, we hypothesize that considering the SA levels when designing AF can reduce the driving errors and thus enhance road safety. To evaluate this hypothesis, we conducted a quantitative study to test the usability of a certain set of feedback and an empirical study using a driving simulator to test the effectiveness of that feedback in terms of improving driving performance, particularly at roundabouts and intersections in an unfamiliar traffic system. The results of the first study enhanced the ability of the in-vehicle information system to provide feedback considering SA levels. This information was incorporated into a driving simulator and provided to drivers. The results of the second study revealed that considering SA levels when designing augmented feedback significantly reduces the driving errors at roundabouts and intersections in an unfamiliar traffic regulation.
\end{abstract}

Keywords-Situation awareness; unfamiliar traffic regulation; augmented feedback; in-vehicle information systems

\section{INTRODUCTION}

International drivers, especially from an opposite, unfamiliar traffic regulation (UFTR), are involved in a large number of traffic accidents [1], [2]. In this paper, a UFTR represents driving a right-hand drive vehicle in a keep-left traffic regulation for drivers who are only familiar with driving a left-hand drive vehicle in a keep-right traffic regulation. The minority of countries (75 countries), such as Australia and Japan [3] are following a UFTR in their traffic system. In the case of UFTR, and especially for international drivers, driving can be more demanding, resulting in an increased number of vehicle accidents, especially at roundabouts and intersections [4].

Overall, satisfactory driving in these conditions requires drivers to be aware of the environment as well as the configurations of the vehicle. This is known as situational awareness (SA). In general, SA refers to the perception of surroundings with respect to time and space, the comprehension of their meaning, and the projection of their status in the future [5]. Accordingly, SA can be divided into three levels [5]: level 1 perception, level 2 comprehension and level 3 projection.

In-vehicle information systems aim to enhance the road safety by providing the drivers with some feedback that help them to make the proper reactions [6]. However, most of existing works ignore designing the provided feedback based on the SA levels, particularly for information required when driving at roundabouts and intersections in a UFTR. The aim of this paper is to consider the SA levels when designing the feedback provided to drivers by in-vehicle information systems. That is, the feedback is provided in perceptual, comprehensible and projectable manner. We hypothesize that considering the SA levels when designing the target feedback can reduce the driving errors and thus improve the road safety. To evaluate this hypothesis, we firstly conducted a quantitative study to test the usability of a certain set of feedback (i.e., travelling path, turning, signalling and speeding at roundabouts and intersections). The results of the first study enhanced the ability of the in-vehicle information system to provide feedback considering SA levels. Then, we conducted an empirical study using a driving simulator to test the effectiveness of that feedback in terms of improving driving performance, particularly at roundabouts and intersections in an unfamiliar traffic system.

The rest of the paper is organized as follows. Section 2 introduces the situation awareness and its levels in driving domain, the rules of driving at roundabouts and intersections in a UFTR and how these rules differ between familiar and unfamiliar traffic regulation, and some in-vehicle information systems that provide visual feedback at roundabouts and intersections. Section 3 tests the usability of presenting the visual feedback in accordance with SA levels. Section 4 evaluates the effectiveness of that feedback in terms of improving the road safety. Section 5 concludes the paper.

\section{BACKGROUND}

\section{A. Situation Awareness in Driving}

$\mathrm{SA}$ in the driving refers to the perception of the elements in the traffic system within a volume of time and space, the comprehension of their meaning, and the projection of their 
status in the near future [7]. Traffic system includes three main components: vehicles on the road, users of the road and road environment. Accordingly, the levels of SA in this domain are: Level 1: Perception of the relevant elements in the traffic system.

Level 2: Comprehension of the meaning of the perceived elements.

Level 3: Projection of near-future status of traffic system.

In relation to driving in a UFTR, the study [8] discovered the drivers' SA when driving at a UFTR by testing the lane changing performance at straight and curved roads in addition to roundabouts and intersections. The findings of [8] showed that international drivers are likely to have low SA when driving at roundabouts and intersections. Accordingly, the current study focuses on driving at roundabouts and intersections in a UFTR.

\section{B. Driving at Roundabouts and Intersections in a UFTR}

Australia is a keep-left country whose traffic system follows a UFTR in this research. In case of no traffic presents in the dual-lane roads, Australian traffic rules are as follows [9]: 1) Approach a roundabout/intersection (a) using the correct approaching lane according to the target direction (i.e. the right, left, and right or left lane when turning right, turning left, and going straight-ahead, respectively), (b) signalling prior to turning left or right and (c) slowing down or completely stopping the vehicle.

2) Enter a roundabout/intersection in the correct direction of traffic flow.

3) Exit a roundabout/intersection using the correct lane.

International drivers who come from keep-right countries must consider the main differences in traffic rules and vehicle configuration when driving at a roundabout/intersection in their home countries and in a UFTR. The differences are as follows:

- In the right-hand drive vehicle (the driver is not familiar with) has its direction indicator stalk on the right of the steering wheel whereas in the left-hand drive vehicle (the driver is familiar with) has its direction indicator stalk on the left of the steering wheel.

- The correct direction when entering a roundabout in a UFTR is clock-wise, not as it is on the keep-right traffic system (i.e. counter clock-wise).

- The correct direction of the traffic flow in a UFTR is the opposite of traffic flow in a keep-right traffic system.

In general, drivers become more aware of the driving environment when they manage to identify the information relevant to the task in rapid patterns to prepare themselves to make appropriate decisions and reactions. This helps the driver understand the relationship between the driving goal and other components of the traffic system at any moment in time [10]. Hence the driver will make fewer errors and avoid vehicle accidents. The information the drivers need can be provided to the drivers by In-vehicle Information Systems (IVISs).

\section{Augmented Feedback in Driving}

Augmented feedback is a form of technology that enhances the performance of a certain task by integrating computergenerated objects with the virtual or real environment [11]. It can be described as a set of stimuli whereby learners receive information from external sources in order to reinforce their behaviour and learning process [12]. The augmented feedback usually targets a task the operator is performing or going to perform in the future. Augmented feedback can be provided in different modalities, such as video, auditory or haptic in order to enrich the user's experience. In general, augmented feedback shows promising results with applications in various human situation, including driving [13], to enhance a number of related physical tasks. In driving, augmented feedback can be provided to drivers via in-vehicle information systems (IVISs).

IVISs are a form of technology that can support drivers by providing real-time feedback returned to drivers telling them what is going. The driver perceives the feedback from the system and understands it to make a decision, and refine or correct his/her driving reactions [14]. That keeps the drivers in the loop and thereby improving the drivers' SA and safety [15]. However, there is growing concern that IVIS might distract drivers from the primary driving task and thus compromise driving safety, as looking at the provided feedback consumes the driver's visual demand. The drivers are required to move their eyes back and forth between the road and the system. Additionally, there is a potential safety issue if the system provides drivers with unnecessary information, as it reduces driving performance [16], [17].

To reach the potential goal of IVISs, the system should provide only the important information. The driver does not need to capture the information that is irrelevant to the current or upcoming driving task. Additionally, the system should provide perceivable information (represents level 1). That is, the driver should not have to take his or her eyes off the road while glancing at the provided information. Also, the system must provide quick comprehensible information [18] (represents level 2). Unclear information might confuse the driver and affect his or her ability to make the correct reaction within the appropriate time. Moreover, the system should provide the information in a projectable manner (represents level 3). That is, the information helps the driver make a correct decision in relation to the upcoming situation. Understanding the drivers' needs of provided information are necessary to design the most useful system.

In relation to driving in a UFTR, the study [8] extracted the required information to drive safe at roundabouts and intersections. The information included upcoming area of the road to inform the driver about the upcoming roundabout and intersection, travelling path to inform the driver about the correct travelling lane when approaching, entering and exiting a roundabout/intersection, direction indicator to remind the driver of the correct position of direction indicator stalk, and speed to inform the driver about the speed limit of the travelling road. Visual feedback is selected by overseas drivers as the most preferable feedback modality to present that information when driving in a UFTR [19]. Therefore, in this research, we only focus on visual feedback provided by IVISs. 


\section{Visual Feedback in Roundabouts/Intersections In-Vehicle Information Systems}

Becic et al., [20] investigated the effectiveness of an IVIS in reducing the number of crashes at a rural stop-controlled intersection in a keep-right traffic regulation. The system displays the upcoming intersection as arrows cross each others on either right or left display based on the traffic flow in the upcoming intersection. The arrows show the traffic direction without showing the required travelling lane the driver should travel within. Also, the information regarding direction indicator and speed limit is not provided to the drivers.

Zhang et al., [21] designed an IVIS that assists the driver when approaching a cross, "arch-shaped" intersection in a keep-left traffic regulation. The system provides visual feedback in a head-up-display (HUP) on the central-lower part of the windshield. The system shows the traffic status of upcoming intersection, leaving the drivers to slow down or make another proper reaction.

Tran et al., [22] studied driving performance at signalised turn-left intersections in a keep-right traffic regulation. In such a driving scenario, the driver does not have priority to turn left. $\mathrm{He} / \mathrm{she}$ must cross the traffic flow coming from the opposite direction. Using a 3D HUP, the IVIS system visualises a three second projected path of the vehicle coming from the opposite side of the road. The path is presented in the driver's main field of view. As long as no vehicle is approaching the intersection, the system presents nothing to the driver. In other words, the system does not describe the situation until traffic exists in the upcoming area.

Caird et al. [23] designed a sign IVIS that visually provides two road signs "prepare to stop" and "signals ahead" on a HUD. These two signs inform the driver of an upcoming change in the road, but they do not provide a complete picture of what is coming next, leaving drivers to guess about whether the traffic light might be at an intersection, roundabout, or Tjunction.

Alyamani et al., 2019 [24] extended the feedback designed in [19] and provided it to the international drivers when driving at a UFTR (see Figure 1). The feedback is presented on a HUD. The feedback includes up-coming section of road, either intersection or roundabout, the correct travelling path, direction indicator and speed limit to help drivers make proper decision and reaction when driving at a roundabout/intersection.

Table I compares different roundabout/intersection invehicle information systems according to feedback the system provides. Two of the works reviewed above support the drivers when driving in a keep-left traffic regulation. All the works reviewed above provide the drivers with feedback related to the upcoming section of the road either prior approaching all upcoming roundabout/intersection or only when traffic exists in the upcoming area. Feedback regarding travelling path is only supported totally in [24] and partially in [22]. None of the above works take into account presenting the direction indicator and speed except [24]. Accordingly, this research focuses on evaluating the usability of the feedback designed by [24] as will be described in the following section.

\section{Testing the Usability of RequiRed Feedback}

A short questionnaire was developed to collect overseas drivers' opinions about the design of the feedback proposed by [24]. We particularly focused on presenting the feedback in a perceivable (level 1), comprehensible (level 2) and projectable (level 3) way. The proposed location of the target feedback was in the lower-central part of the windshield, using a HUD (level 1), the content included well-known symbols, icons and road signs (level 2) to help the driver make decisions about the required driving performance and behaviour (level 3).

\section{A. Questionnaire Design and Procedure}

An open recruitment process was adopted. Participants were recruited via an email that included the survey link. At the beginning of the questionnaire, information and instructions regarding driving at roundabouts and intersections in NSW, Australia, were presented. The questionnaire started by collecting demographic information, such as gender, age and driving experience in both keep-right and keep-left traffic regulations. Then, a GIF image of a vehicle driving in a simulated keep-left road was presented to give participants a better sense of driving in a keep-left traffic regulation, especially for those who were only familiar with a keep-right traffic regulation. Then, the participants were asked to answer the usability questions. Table II shows the list of questions, the target feedback and the rating scale. Each question included an image (see Fig. 2); participants were asked to observe the image and answer the question. The participants could add any comments after answering the question. Questions QA, QBs and QCs addressed the usability of the information in relation to its perceivability (level 1), comprehensibility (level 2) and projectability (level 3 ), respectively.

\section{B. Participants}

We distributed the online surveys to 65 participants, aged 19-52 with mean of 31.7 (SD: 1.1). The participants were only familiar with a keep-right regulation. All had a driver's license issued in their home keep-right country with a mean driving experience of 13.5 years $(\mathrm{SD}=1.3)$ and drove in familiar regulations for an average of 19.7 hours/week $(\mathrm{SD}=1.9)$.

\section{Data Analysis}

The usability questions were grouped into two categorical variables. Responses of the first question were categorised as 'easy', (including 'easy' and 'very easy') and 'difficult' (including 'very difficult' and 'difficult'). Responses to the second question were categorised as 'clear' (which included 'very clear' and 'clear') and 'not clear' (including 'not very clear' and 'not clear at all'). Responses to the third question were grouped into 'useful', (including 'very useful' and 'useful') and 'not useful' (including 'not very useful' and 'not useful at all'). Undecided responses were excluded. As we had one sample and categorical variables, we conducted a nonparametric test (i.e. one sample chi-square goodness-of-fit test) [25] to determine usability in relation to each question. 


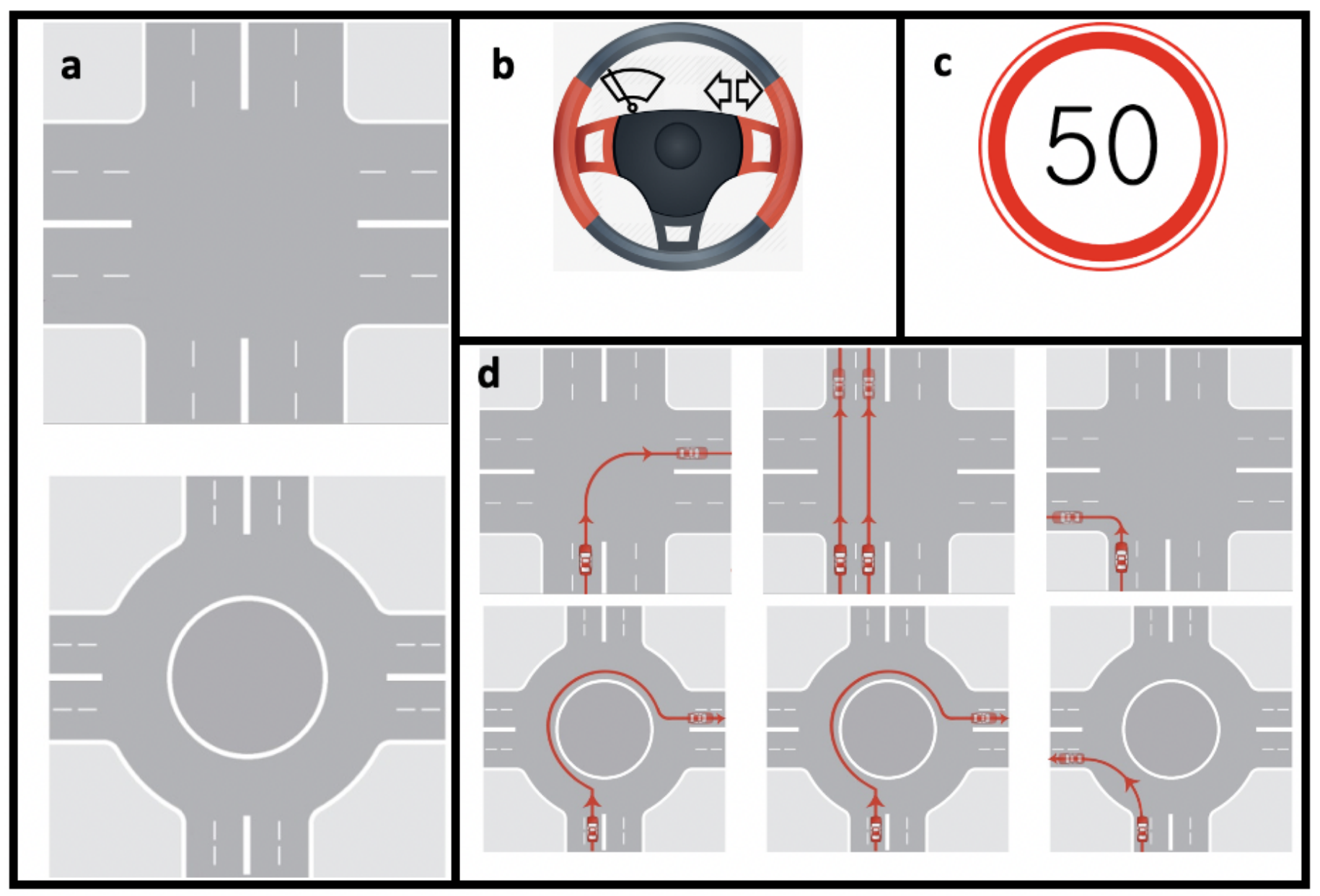

Fig. 1. Extended Design of Required Feedback to Support Overseas Drivers when Driving in Roundabouts and Intersections [24]. The Feedback Include a) the Upcoming Section of the Road, b) using the Direction Indicator,c) Speed Limit and d) Travelling Path).

TABLE I. OVERVIEW of A ROUNDABOUt/INTERSECTION IVIS RELATED to TRAFFIC REGULATION AND REQUIRED FEEDBACK. • : SUPPORTED, o: NOT

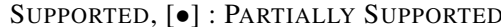

\begin{tabular}{|l|l|l|l|l|l|}
\hline Authors & Traffic regulation & Upcoming section of road & Travelling & Using direction indicator & Speed limit \\
\hline Becic et al. 2012 [20], & KRT & $\bullet$ & $\circ$ & $\circ$ & $\circ$ \\
Zhang et al. 2009 [21], & KLT & $\bullet$ & $\circ$ & $\circ$ & $\circ$ \\
Tran et al. 2013 [22] & KRT & {$[\bullet]$} & {$[\bullet]$} & $\circ$ & $\circ$ \\
Caird et al., 2008 [23] & KRT & $\bullet$ & $\circ$ & $\circ$ & $\circ$ \\
Alyamani et al., 2019 [24] & KLT & $\bullet$ & $\bullet$ & $\bullet$ & $\bullet$ \\
\hline
\end{tabular}

\section{Results}

In relation to the first question, 44 participants thought feedback presented on the lower-central part of the windshield using a HUD was easy to perceive whereas 10 participants thought it was difficult (see Table III). The minimum expected frequency was 27.0. The difference was statistically significant $\left(\chi^{2}(1)=21.407, \rho<.001\right)$.

The majority of participants (55) thought the upcoming area feedback clearly described the conditions of the upcoming area; only 2 participants did not think it was clearly described. The minimum expected frequency was 25.5. The difference was statistically significant, $\chi^{2}(1)=49.281, \rho<.001$. On the other hand, 27 participants thought the upcoming area feedback could help them make a decision regarding the required reaction or performance at the upcoming roundabout or intersection, while 24 participants thought this feedback could not help them do so. The minimum expected frequency was 25.5 . The difference was not statistical significant $\left(\chi^{2}(1)=.176, \rho=.674\right)$.

Most participants (56) thought that the combination of upcoming area and travelling path feedback clearly described the situation; only 3 participants found that this combination did not clearly describe the situation. The minimum expected frequency was 29.5 . The difference was statistically $\operatorname{significant}\left(\chi^{2}(1)=47.610, \rho<.001\right)$. Similarly, 59 participants thought that presenting upcoming area and travelling path feedback was useful for planning the required reaction at the upcoming section of road; only 2 participants thought it was not useful. The minimum expected frequency was 30.5 . The difference was statistically significant $\left(\chi^{2}(1)=53.262, \rho<\right.$ 
TABLE II. QUestions to Evaluate the Usability of NeCessary FeEdBack

\begin{tabular}{|c|c|c|c|}
\hline Q\# & Question & Target feedback & Rating scale \\
\hline QA & How easy is it to perceive the feedback in the image below? & All & $\begin{array}{l}\text { 'very difficult', 'difficult', } \\
\text { 'normal', 'easy' and 'very easy' }\end{array}$ \\
\hline QB1 & $\begin{array}{l}\text { How well does the feedback describe the situation shown } \\
\text { in the clip above? }\end{array}$ & Upcoming area & $\begin{array}{l}\text { 'not clear at all', 'not very clear', } \\
\text { 'normal', 'clear', 'very clear' }\end{array}$ \\
\hline QB2 & $\begin{array}{l}\text { How well does the feedback describe the situation shown } \\
\text { in the clip above? }\end{array}$ & $\begin{array}{l}\text { Upcoming area }+ \\
\text { travelling path }\end{array}$ & \\
\hline QB3 & $\begin{array}{l}\text { How well does the feedback describe the situation shown } \\
\text { in the clip above? }\end{array}$ & $\begin{array}{l}\text { Upcoming area }+ \\
\text { direction indicator }\end{array}$ & \\
\hline QB4 & $\begin{array}{l}\text { How well does the feedback describe the situation shown } \\
\text { in the clip above? }\end{array}$ & $\begin{array}{l}\text { Upcoming area }+ \\
\text { speeding }\end{array}$ & \\
\hline$\overline{\mathrm{QC} 1}$ & $\begin{array}{l}\text { How useful is the feedback helping you make decisions } \\
\text { about the required performance at the upcoming } \\
\text { roundabout/intersection? }\end{array}$ & Upcoming area & $\begin{array}{l}\text { 'not useful at all', 'not very } \\
\text { useful', 'normal', 'useful', } \\
\text { 'very useful' }\end{array}$ \\
\hline QC2 & $\begin{array}{l}\text { How useful is the feedback helping you make decisions } \\
\text { about the required performance at the upcoming } \\
\text { roundabout/intersection? }\end{array}$ & $\begin{array}{l}\text { Upcoming area }+ \\
\text { travelling path }\end{array}$ & \\
\hline QC3 & $\begin{array}{l}\text { How useful is the feedback helping you make decisions } \\
\text { about the required performance at the upcoming } \\
\text { roundabout/intersection? }\end{array}$ & $\begin{array}{l}\text { Upcoming area }+ \\
\text { direction indicator }\end{array}$ & \\
\hline QC4 & $\begin{array}{l}\text { How useful is the feedback helping you make decisions } \\
\text { about the required performance at the upcoming } \\
\text { roundabout/intersection? }\end{array}$ & $\begin{array}{l}\text { Upcoming area }+ \\
\text { speeding }\end{array}$ & \\
\hline
\end{tabular}
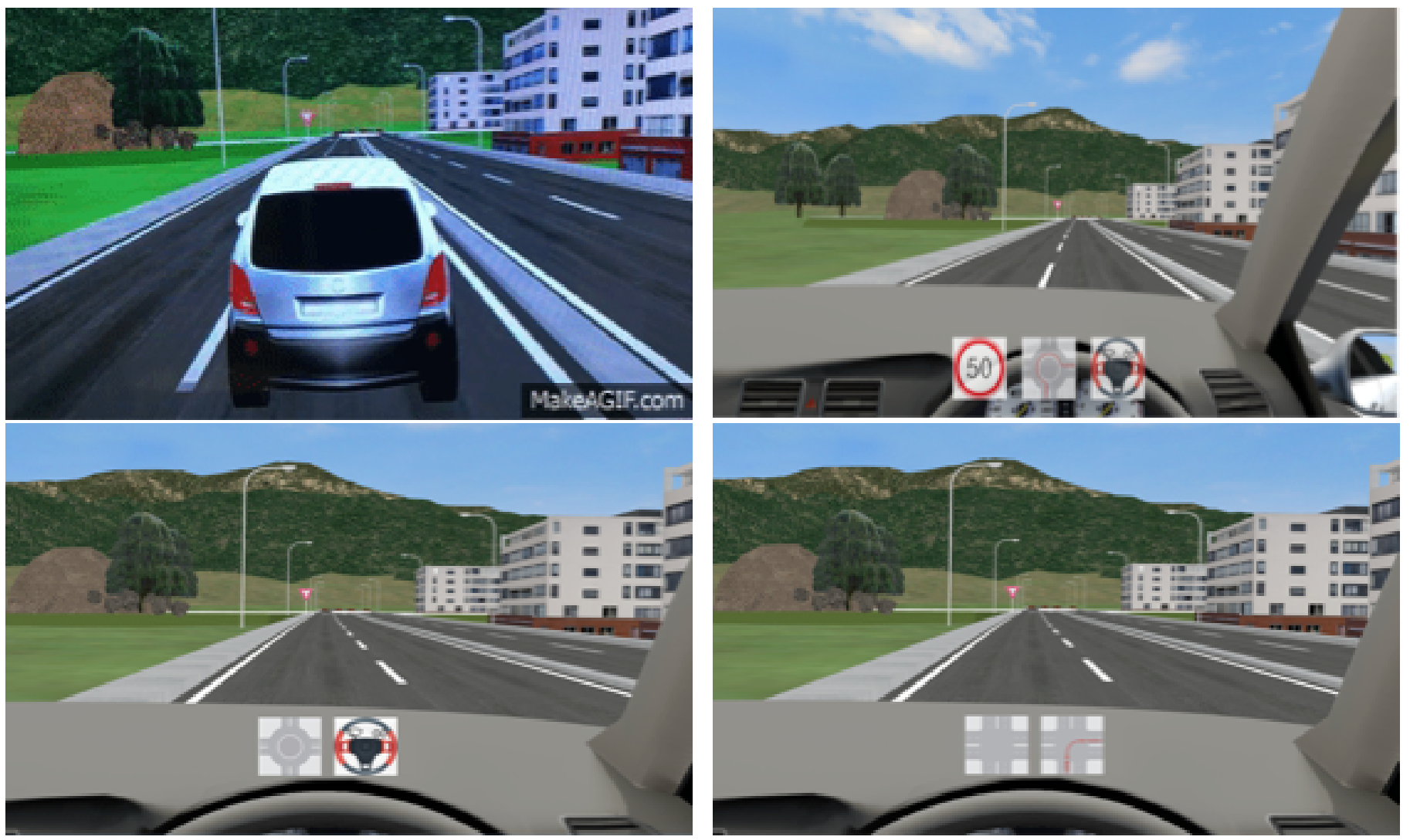

Fig. 2. Some Images used in the Usability Questionnaire. 
TABLE III. Positive AND Negative RATINGS For EACH QUeStion

\begin{tabular}{|l|l|l|c|c|}
\hline Question & \# of 'easy' responses & \# of 'difficult' responses & $\chi^{2}(1)$ & $\mathrm{p}$ \\
\hline QA & 44 & 10 & 21.407 & $<.001^{*}$ \\
\hline \hline Question & \# of 'clear' responses & \# of 'not clear' responses & $\chi^{2}(1)$ & $\mathrm{p}$ \\
\hline QB1 & 55 & 2 & 49.281 & $<.001^{*}$ \\
QB2 & 56 & 3 & 47.610 & $<.001^{*}$ \\
QB3 & 47 & 10 & 24.018 & $<.001^{*}$ \\
QB4 & 57 & 5 & 43.613 & $<.001^{*}$ \\
\hline \hline Question & \# of 'useful' responses & \# of 'not useful' & $\chi^{2}(1)$ & $\mathrm{p}$ \\
\hline QC1 & 27 & 24 & .176 & $=.674$ \\
QC2 & 59 & 2 & 53.262 & $<.001^{*}$ \\
QC3 & 39 & 12 & 14.294 & $<.001^{*}$ \\
QC4 & 62 & 0 & - & - \\
\hline
\end{tabular}

* Statistically significant.

$.001)$.

In relation to upcoming area and direction indicator feedback, 47 participants thought this combination clearly described the situation, whereas 10 participants thought it did not. The minimum expected frequency was 28.5. The difference was statistically significant, $\chi^{2}(1)=24.018, \rho<.001$. In addition 39 participants thought it was useful to plan the required reaction using both upcoming area and direction indicator feedback together, whereas 12 participants thought it was not useful to do so. The minimum expected frequency was 25.5 . The difference was statistically significant $\left(\chi^{2}(1)=\right.$ $14.294, \rho<.001)$.

In relation to a combination of upcoming area and speeding feedback, 57 participants thought presenting upcoming area and speeding feedback together clearly described the situation, while 5 participants thought it did not. The minimum expected frequency was 31.0. The difference was statistically significant $\left(\chi^{2}(1)=43.613, \rho<.001\right) .62$ participants thought it was useful to plan the required reaction using that combination of feedback; no participant thought it was not useful to do so. The chi-square goodness-of-fit test could not be performed as the variable was constant (there was no value for the 'not useful' group).

\section{E. Discussion}

A significant percentage of participants $(67.7 \%)$ reported that feedback displayed on a HUD was easy to perceive. Therefore, we should present the target feedback on a HUD. A significant percentage of participants $(84.6 \%)$ also thought that providing only upcoming area feedback (i.e. static feedback) clearly described the upcoming situation. However, there was insignificant difference between the percentage of participants $(41.5 \%)$ who reported that upcoming area feedback was useful for directing the driver to make the proper decisions at the upcoming section of road and those who did not (36.9\%).

Participants were confused about the required reaction due to their unfamiliarity with the keep-left traffic regulation, even the driving rules at roundabouts and intersections were explained at the beginning of the questionnaire. On the other hand, a significant percentage of participants thought that combining upcoming area information with any of the other forms of dynamic feedback (i.e. travelling path, direction indicator and speed) clearly described the situation and was useful for making appropriate decisions at an upcoming section of road. In fact, $86.2 \%, 72.3 \%$ and $87.7 \%$ of participants found that the combination of upcoming area and travelling path, upcoming area and direction indicator, upcoming area and speed, respectively, clearly described the situation of the upcoming section of road. Also, $90.8 \%, 60.0 \%$ and $95.4 \%$ of participants found the combination of upcoming area and travelling path, upcoming area and direction indicator, upcoming area and speed, respectively, was useful for making appropriate decisions at an upcoming section of road. Hence, we recommend providing static feedback supported by dynamic feedback. The static feedback functioned to describe provided the upcoming situation while dynamic feedback supported decision making.

In relation to a combination of upcoming area and travelling path feedback, four participants $(6.2 \%)$ who responded that this combination was clear and useful recommended that we use only travelling path instead, as travelling path was shown on the roundabout and intersection. Two participants $(3.1 \%)$ who were undecided about the clarity and usefulness of this combination found the simultaneous presentation confusing. One participant $(1.5 \%)$ who had a negative response regarding the clarity and usefulness of that combination commented: "I do not know if I will drive through one or two roundabouts" and "should I follow the line at first and second roundabout?" As a result of the wider potential for such confusion, we would follow the recommendations we received from participants and only provide travelling path feedback when it was necessary.

\section{EFFeCtiveness Evaluation}

The study evaluates the effectiveness of the feedback mentioned above for improving the road safety, particularly for drivers who are not familiar with driving in a UFTR.

\section{A. Method}

1) Participants: The study involved twenty international participants. The participant age range was 20-35 years with a mean age of 24.2 years $(S D=2.7)$. All participants were unfamiliar with a keep-left traffic regulation and right-hand drive vehicle. They each had a driving license of their home country with a mean driving experience of 5.9 years $(S D=$ $3.3)$. They drove in average 14.7 hours/week $(S D=9.0)$.

2) Apparatus: The experiment was conducted on the Forum8 drive simulator, which is a fixed-based simulator. It represents a right-hand drive vehicle with three 42 -inch monitors, creating a 150 degree horizontal and 30 degree vertical field of view. The monitors display the central and peripheral visual 
fields to the driver. UC-win/Road was used as software to model the traffic system in addition to locating the visual feedback on of the monitors. The driving data (e.g., speed, brake and steering wheel angel) was recorded for future analysis.

3) Driving Track, Scenario and Tasks: Using Forum8, the participants drove in a simulated keep-left traffic system (see Fig. 3- (a)). The driving track was similar to the track designed in [24]. The track included dual-lane roads crossed each other in three roundabouts and intersections. Participants were asked to drive in a certain direction, which forced them to drive through all roundabouts and intersections, each time with a different direction (left, right, and forward). Participants were asked to drive appropriately at those roundabouts and intersections following the Australian rules (see Section II-B).

4) Procedure: The experiment had three sessions - preexperiment, preparation and two driving tests. In the preexperiment session, participants were asked to complete an initial computer-based questionnaire. It was used to collect demographic information and driving experience data. In the preparation session, the driving simulator, Forum8, was introduced. Participants were verbally informed about the upcoming session and the Australian traffic rules, focusing on the rules for driving at roundabouts and intersections. In addition, they received a quick explanation of differences in vehicle configuration, such as the position of the wiper and direction indicator stalk, whereas the direction indicator stalk of a right-hand drive vehicle is placed the other way around. Then, participants were driving in a built-in test scenario for 10 minutes in order to familiarise themselves with the driving simulator. This scenario was supported by Forum8 and represented a keep-left driving environment. The scenario followed a route that differed from the test route to minimise the learning effect. During the familiarisation test, the researcher answered any questions participants might have. Participants had a 5minute break after the familiarisation test while the researcher prepared the simulation for the driving test.

The third session (i.e. driving tests) had two driving trials - "Control" (no feedback offered) and "Experimental", (providing feedback). The researcher randomly divided the 20 participants into two similar sized groups (10 participants each). Both groups participated in both trials. However, the order of the two trials was randomised between them in order to reduce the learning effect. Whereas the first group started with the control trial, the second group started with the experimental trial. There was a one-hour break between the two trials. Each driving test took around seven minutes to complete. The session started with providing the participants with a map of the driving track that indicated the direction the driver should follow (see Fig. 3- (b)). Participants took approximately three minutes to study the map. Then, they were asked to complete the driving tasks of each trial without making driving errors. In the experimental trial, participants did not receive any information regarding the feedback they would receive.

5) Data Collection and Analysis: Two log files (video and CSV) were generated by the simulator for each participant and for each trial. Both log files had data on driving performance. For each trial, we observed the following driving errors:
Error 1 Driving in an incorrect lane when approaching the roundabout and the intersection.

Error 2 Not indicating the target direction when approaching the roundabout and the intersection.

Error 3 Speeding while approaching the roundabout and the intersection.

Error 4 Driving in the incorrect direction of traffic flow inside the roundabout/intersection.

Error 5 Driving in an incorrect lane when exiting the roundabout and the intersection.

The number of the above errors that occurred at all roundabouts and intersections was calculated. The Wilcoxon signed-rank test was run to compare the number of each error in the experimental trial versus the control trial.

\section{B. Results}

Overall, the total number of errors among participant of each error type decreased when the feedback was provided. The difference in number of each driving error with and without providing feedback among participants was not normally distributed (see Table IV). For instance, the difference of driving in an incorrect lane when exiting the roundabout/intersection (Error 3) had a skewness of 1.032 (0.512) and a kurtosis of $-0.230(0.992)$. Thus, a non-parametric test (i.e. Wilcoxon signed-rank test) was employed to investigate differences between the driving errors before and after providing feedback.

Wilcoxon signed rank test (see Table V) showed that the numbers of Error 1, Error 2, Error 3 and Error 4 when providing augmented feedback were statically significantly fewer than than the numbers of those errors when augmented feedback was not provided $(Z=-2.648, p=.008)$, $(Z=-1.998, p=.046),(Z=-2.167, p=.030)$, and ( $Z=-2.266, p=.023$ ), respectively. On the other hand, Wilcoxon signed rank test indicated that the number of Error 5 when providing augmented feedback was insignificant fewer than the number of Error 5 when the augmented feedback was not provided to participants $(Z=-1.642, p=.101$.).

\section{Discussion}

In general, presenting perceivable, comprehensible and projectable feedback significantly assisted the drivers who are not familiar with driving in a keep-left regelation to approach and enter roundabouts and intersections in a UFTR.

One participant mentioned indicating the target direction was very difficult as the direction indicator stalk is located on the right side of the steering wheel not on the left as it is on the vehicle that he used to drive in my home country. However, presenting perceivable, comprehensible and projectable direction indicator feedback significantly reduced the number of using improver direction indicators when approaching roundabouts and intersections $(Z=-1.998, p=.046)$. One participant commented: "it was easier for me to look at the indicator feedback instead of looking at the real stalks to find out which is the correct stalk to use, the right or the left". Another participant mentioned that direction indicator feedback corrected him when he used the wiper indicator instead of direction indicator. 


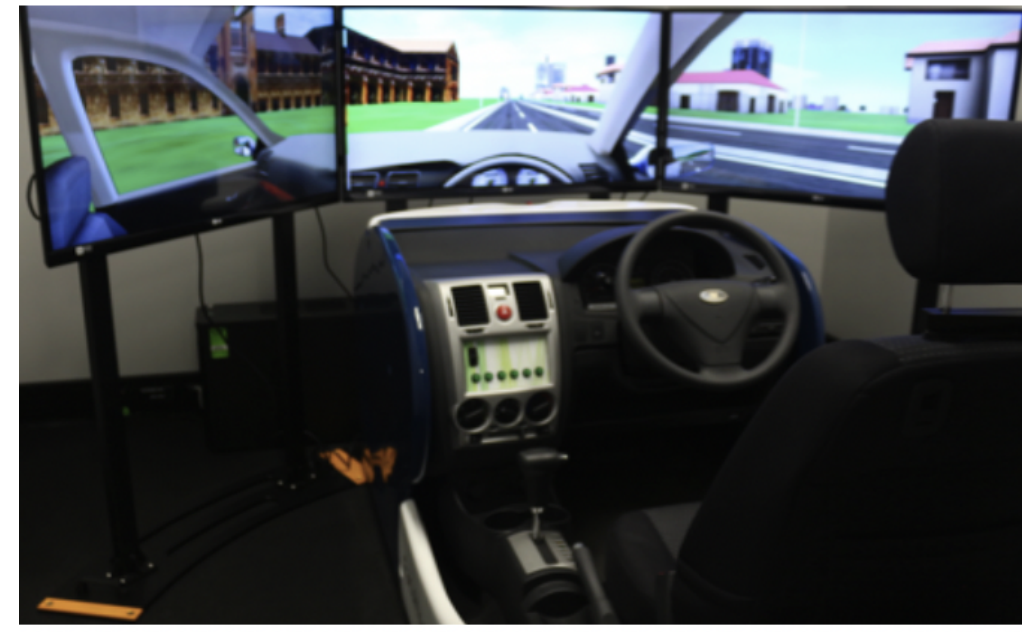

a

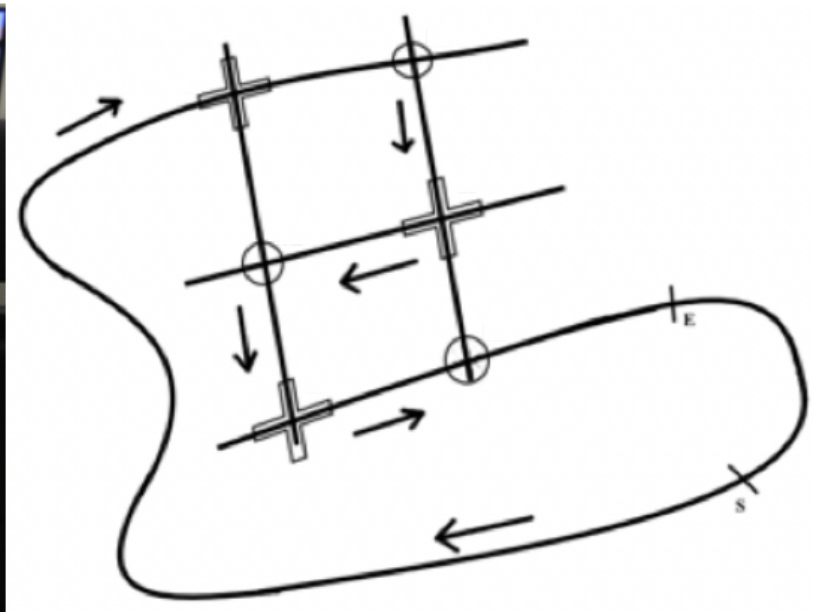

b

Fig. 3. (a) Forum8 (b) The Start and End Points and Required Direction on the Driving Track. Adapted from [24].

TABle IV. Results of Normality Tests for the Difference in Number of Each ERror Type with and without Feedback

\begin{tabular}{|l|l|l||l|l|l|}
\hline Error & Shows (SE) & Kurtosis (SE) & Error & Shows (SE) & Kurtosis (SE) \\
\hline Error 1 & $-2.573(0.512)$ & $9.386(0.992)$ & Error 4 & $1.032(0.512)$ & $-0.230(0.992)$ \\
\hline Error 2 & $0.992(0.512)$ & $1.712(0.992)$ & Error 5 & $-0.120(0.512)$ & $-0.088(0.992)$ \\
\hline Error 3 & $0.991(0.512)$ & $0.263(0.992)$ & & & \\
\hline
\end{tabular}

TABLE V. Number of Driving ERrors Grouped by ERror Type with AND without FeEdback Support

\begin{tabular}{|l|c|c|c|c|c|}
\hline Error & Number of errors (Control trial) & Number of errors (Experimental trial) & Difference & $\mathrm{Z}$ & $\mathrm{p}$ \\
\hline Error 1 & 20 & 5 & -15 & -2.648 & $.008^{*}$ \\
Error 2 & 17 & 11 & -6 & -1.998 & $.046^{*}$ \\
Error 3 & 34 & 18 & -16 & -2.167 & $.030^{*}$ \\
Error 4 & 15 & 1 & -14 & -2.266 & $.023^{*}$ \\
Error 5 & 39 & 28 & -11 & -1.642 & .101 \\
\hline
\end{tabular}

* Statistically significant.

The correct direction when entering a roundabout in a UFTR is clock-wise, not as it is on the keep-right traffic system. In addition, the correct direction when entering an intersection in a UFTR is the opposite side of the road that the driver is familiar to use when driving in a keep-right traffic system. That might lead the drivers to make improper decision when approaching roundabouts and intersections in a UFTR. For instance, in case of approaching a roundabout, if the driver does not realise those changes in traffic rules, the driver might approach the roundabout using improper travelling lane. Also, the driver might enter the roundabout from the wrong direction. However, presenting perceivable, comprehensible and projectable travelling path significantly reduced the cases of approaching the roundabouts and intersections from incorrect lane $(Z=-2.648, p=.008)$ and entering roundabouts and intersections from the incorrect direction $(Z=-2.266$, $p=.023)$. One participant mentioned that travelling path feedback was very clear and understandable and that helped him to recognize the correct travelling path.

On the other hand, controlling the speed of the vehicle is a task that is not changed when driving in a familiar and unfamiliar traffic regulation. That is, the driver does not require to adapt his or her speeding behaviour when driving in a UFTR. However, presenting perceivable, well-known and pro- jectable speeding feedback helped the drivers to significantly reduce their speed when approaching the roundabouts and intersections $(Z=-2.167, p=.030)$. This result might be influenced by providing other information next to speed feedback. Other studies [20], [21], [22], [23] managed to reduce the drivers' speed without presenting speed feedback. More research is needed to explore the exact feedback that assisted the drivers to slowdown.

\section{CONCLUSION}

In this paper, we presented the results of a quantitative study that subjectively investigated the usability of augmented visual feedback that designed to help the drivers when driving at roundabouts and intersections in an unfamiliar traffic regulation (i.e. a keep-left traffic regulation using a keepright drive vehicle). The usability was evaluated based on the situation awareness levels. That is, the information should be designed in a perceivable, comprehensible and projectable manner. Sixty-five participants who were not familiar with an Australian traffic regulation answered the online survey. The results enhanced the ability of the driver to capture the provided information, understand it and plan appropriately for the required driving reaction.

Then, another twenty participants who were not familiar 
with an Australian traffic regulation participated in an empirical study. The participants drove with and without providing the feedback in a simulated keep-left traffic environment using a driving simulator (i.e. Forum8). The study aimed to evaluate the effectiveness of the perceivable, comprehensible and projectable feedback in reducing the driving errors and thus improving the road safety. The results confirmed our hypothesis that considering the SA levels when designing the feedback can significantly reduce the driving errors when reaching and entering roundabouts and intersections and thus enhance road safety in a UFTR.

The current work focused mainly on driving at roundabouts and intersections in a UFTR. Thus, the work was limited by feedback that provided for a particular driving task. Another limitation was that presenting the visual feedback in accordance with SA levels was evaluated in a specific driving condition (a fine weather with no traffic movement).

The current work can be extended to cover the following points:

- Evaluate the usability of further feedback that designed to assist the drivers in different driving tasks, such as lane-changing and parking.

- Evaluate the effectiveness of the feedback in other driving conditions, such as rainy and traffic jam.

\section{REFERENCES}

[1] S. J. Page and D. Meyer, "Tourist accidents: an exploratory analysis," Annals of Tourism Research, vol. 23, no. 3, pp. 666-690, 1996.

[2] J. Wilks, B. Watson, and J. Hansen, "International drivers and road safety in queensland, australia," Journal of Tourism Studies, vol. 11, no. 2, pp. 36-43, 2000.

[3] "Which countries drive on the left - a handy guide!" 2017. [Online]. Available: https://www.rhinocarhire.com/CustomerServices/Privacy-Policy.aspx

[4] Ministry of Transport, "Overseas driver crashes (including matched crash and visitor arrival data)," Ministry of Transport, Wellington, New Zealand, Tech. Rep., 2016.

[5] M. R. Endsley, "Situation awareness global assessment technique (sagat)," in Proceedings of the IEEE 1988 National Aerospace and Electronics Conference, vol. 3, 1988, pp. 789-795.

[6] J. Piao and M. McDonald, "Advanced driver assistance systems from autonomous to cooperative approach," Transport reviews, vol. 28, no. 5, pp. 659-684, 2008

[7] L. Gugerty et al., "Situation awareness in driving," Handbook for driving simulation in engineering, medicine and psychology, vol. 1, pp. 265-272, 2011.

[8] H. J. Alyamani and M. Kavakli, "Situational awareness and systems for driver-assistance," in Proceedings of the 50th Hawaii International Conference on System Sciences, 2017.

[9] NSW Roads and Maritime Services, "Road users' handbook," 2015.

[10] N. A. Stanton, A. Dunoyer, and A. Leatherland, "Detection of new inpath targets by drivers using stop \& go adaptive cruise control," Applied ergonomics, vol. 42, no. 4, pp. 592-601, 2011.
[11] R. Sigrist, G. Rauter, R. Riener, and P. Wolf, "Augmented visual, auditory, haptic, and multimodal feedback in motor learning: a review," Psychonomic bulletin \& review, vol. 20, no. 1, pp. 21-53, 2013.

[12] R. Schmidt and C. Wrisberg, Motor learning and performance: A problem-based learning approach, third edition ed. Champaign, IL: Human Kinetics, 2004.

[13] S. De Groot, J. C. De Winter, J. M. L. García, M. Mulder, and P. A. Wieringa, "The effect of concurrent bandwidth feedback on learning the lane-keeping task in a driving simulator," Human factors, vol. 53, no. 1, pp. 50-62, 2011.

[14] N. A. Stanton and P. M. Salmon, "Human error taxonomies applied to driving: A generic driver error taxonomy and its implications for intelligent transport systems," Safety Science, vol. 47, no. 2, pp. 227237, 2009.

[15] T. O. Heijer, H.-1. Wiethoff, M. Boverie, S. Penttinen, M. Schirokoff, A. Kulmala, R. Heinrich, J. Ernst, A. Sneek, and N. Heeren, "Action for advanced drivers assistance and vehicle control system implementation, standardisation, optimum use of the road network and safety advisors deliverable d1/2.1 v1: Problem identification, user needs and inventory of adas (advanced driver assistance systems): Final report," Contract No. DGTREN GRD1 2000-10047, Commission of the European Communities, Directorate-General for Energy and Transport, Brussels, Tech. Rep., 2002.

[16] P. Dalton, P. Agarwal, N. Fraenkel, J. Baichoo, and A. Masry, "Driving with navigational instructions: Investigating user behaviour and performance," Accident Analysis \& Prevention, vol. 50, pp. 298-303, 2013.

[17] J. B. Van Erp and H. A. Van Veen, "Vibrotactile in-vehicle navigation system," Transportation Research Part F: Traffic Psychology and Behaviour, vol. 7, no. 4, pp. 247-256, 2004.

[18] M. Baumann, A. Keinath, J. F. Krems, and K. Bengler, "Evaluation of in-vehicle hmi using occlusion techniques: experimental results and practical implications," Applied ergonomics, vol. 35, no. 3, pp. 197-205, 2004.

[19] H. J. Alyamani, A. Hinze, S. Smith, and M. Kavakli, "Preference feedback for driving in an unfamiliar traffic regulation," in Service Research and Innovation. Springer, 2018, pp. 35-49.

[20] E. Becic, M. P. Manser, J. I. Creaser, and M. Donath, "ntersection crossing assist system: Transition from a road-side to an in-vehicle system," Transportation research part F: traffic psychology and behaviour, vol. 15, no. 5, pp. 544-555, 2012.

[21] J. Zhang, K. Suto, and A. Fujiwara, "Effects of in-vehicle warning information on drivers' decelerating and accelerating behaviors near an arch-shaped intersection," Accident Analysis \& Prevention, vol. 41, no. 5, pp. 948-958, 2009.

[22] C. Tran, K. Bark, and V. Ng-Thow-Hing, "A left-turn driving aid using projected oncoming vehicle paths with augmented reality," in Proceedings of the 5th International Conference on Automotive User Interfaces and Interactive Vehicular Applications. ACM, 2013, pp. 300-307.

[23] J. Caird, S. Chisholm, and J. Lockhart, "Do in-vehicle advanced signs enhance older and younger drivers' intersection performance? driving simulation and eye movement results," International journal of humancomputer studies, vol. 66, no. 3, pp. 132-144, 2008.

[24] H. J. Alyamani, M. Kavakli, and S. Smith, "Vehand: an in-vehicle information system to improve driving performance in an unfamiliar traffic regulation," International journal of human factors and ergonomics, vol. 6, no. 4, pp. 355-389, 2019.

[25] N. L. Leech, K. C. Barrett, and G. A. Morgan, SPSS for intermediate statistics: Use and interpretation. Psychology Press, 2005. 